\title{
Identification of coding and non-coding mutational hotspots in cancer genomes
}

Scott W. Piraino ${ }^{1}$ and Simon J. Furney ${ }^{2^{*}}$

\begin{abstract}
Background: The identification of mutations that play a causal role in tumour development, so called "driver" mutations, is of critical importance for understanding how cancers form and how they might be treated. Several large cancer sequencing projects have identified genes that are recurrently mutated in cancer patients, suggesting a role in tumourigenesis. While the landscape of coding drivers has been extensively studied and many of the most prominent driver genes are well characterised, comparatively less is known about the role of mutations in the non-coding regions of the genome in cancer development. The continuing fall in genome sequencing costs has resulted in a concomitant increase in the number of cancer whole genome sequences being produced, facilitating systematic interrogation of both the coding and non-coding regions of cancer genomes.

Results: To examine the mutational landscapes of tumour genomes we have developed a novel method to identify mutational hotspots in tumour genomes using both mutational data and information on evolutionary conservation. We have applied our methodology to over 1300 whole cancer genomes and show that it identifies prominent coding and non-coding regions that are known or highly suspected to play a role in cancer. Importantly, we applied our method to the entire genome, rather than relying on predefined annotations (e.g. promoter regions) and we highlight recurrently mutated regions that may have resulted from increased exposure to mutational processes rather than selection, some of which have been identified previously as targets of selection. Finally, we implicate several pan-cancer and cancer-specific candidate non-coding regions, which could be involved in tumourigenesis.

Conclusions: We have developed a framework to identify mutational hotspots in cancer genomes, which is applicable to the entire genome. This framework identifies known and novel coding and non-coding mutional hotspots and can be used to differentiate candidate driver regions from likely passenger regions susceptible to somatic mutation.
\end{abstract}

Keywords: Cancer genome sequencing, Non-coding mutations, Mutational hotspots

\section{Background}

The characterisation of driver mutations in tumour genomes is a major component of cancer genomics research [1-3]. Cancer develops when somatic cells sustain genetic damage. Some mutations generated in this manner allow a cell and its progeny to survive and divide more rapidly, eventually generating a detectable tumour. However, a large fraction of mutations present in cancer genomes do not confer a detectable advantage to cells, therefore do not experience somatic selection and are termed passenger mutations. The mutations that do confer an advantage

\footnotetext{
* Correspondence: simon.furney@ucd.ie

${ }^{2}$ School of Biomolecular and Biomedical Science, Conway Institute of Biomolecular and Biomedical Research, University College Dublin, Belfield, Dublin 4, Ireland

Full list of author information is available at the end of the article
}

to cancerous cells are positively selected during tumour development, and are referred to as driver mutations [4]. Driver mutations are causally related to the development of individual cancers, so cataloging potential driver mutations is critical to understanding the mechanisms and dynamics of tumour development. Additionally, because driver mutations contribute to and sometimes are essential for the growth and survival of a tumour, the presence or absence of specific driver mutations are strong candidate biomarkers for personalized cancer therapies.

Driver mutations within the coding regions of the genome have been extensively characterized [4-8]. This has generally taken the form of large studies both within and across cancer types that have attempted to identify driver genes (genes that contain driver mutations). As a result of this work, several strategies have been developed that can 
be used to infer regions that are targets of positive somatic selection (putative driver regions) from the somatic mutations present in large sets of tumours. Positive selection is expected to increase the frequency with which a mutation is observed in sequencing experiments above the rate expected simply from mutational processes alone. As a result, recurrence of a mutation, or mutations within a given region of the genome relative to the mutation rate of that region is a signal of positive selection [6-9]. Driver mutations are also likely to be mutations that have strong functional effects. As a result, the functional consequence of a mutation can be an indication of the likelihood that a mutation or region has driver potential [10]. In the context of coding mutations for example, nonsynonymous mutations are apriori more likely to be driver mutations than synonymous mutations. Driver mutations often display a clustered pattern within driver regions across tumours, particularly in oncogenes $[11,12]$. This can be the case when mutations in two separate tumours target the same functional site or domain, creating a clustered pattern where mutations tend to occur within the same region, and are mutually exclusive across individual tumours (i.e. only one mutation at the site per tumour).

Most efforts to characterize driver mutations have focused exclusively on coding regions of the genome, but recent examples of non-coding mutations that can contribute to tumourigenesis have sparked interest in the non-coding regions of the cancer genome [13]. For example, mutations in the promoter of the telomerase reverse transcriptase (TERT) gene have been identified as pan-cancer driver mutations that function by creation of a de novo transcription factor binding site upstream of TERT, resulting in TERT mRNA upregulation $[14,15]$. TERT mutations occur recurrently at two nucleotides upstream of TERT in a mutually exclusive manner. Several studies have also conducted systematical screens of the non-coding regions of the genome for driver mutations [16-25]. These efforts have mainly focused on identifying recurrently mutated regions, but have also included other approaches. In the context of non-coding mutations, one potential strategy is to use various annotations to increase the priority given to certain types of mutations, similar to the use of annotations (e.g. PolyPhen, SIFT) for coding mutations. Examples of annotations that have been applied to non-coding mutations include information about motif disruption/creation $[19,21,24]$ and human germline polymorphism frequency [19]. Other studies have correlated non-coding mutation status with mRNA expression $[18,21]$ and clinical data $[21,26]$.

These studies have predominantly focused on the subset of the non-coding genome that is most likely to be functional (e.g. promoter or regulatory regions). However, there may be driver regions that lie outside of currently known functional regions or in less well-documented and studied regions. As such, the aforementioned studies notwithstanding, the extent and significance of the contribution of non-coding mutations in cancer development has yet to be fully eludicated. This is in part due to the fact that we do not possess a clear appreciation of how to extricate the information from cancer genomes necessary to interpret the significance of non-coding mutations.

Therefore, in this study we sought to develop a novel method for the identification of mutational hotspots in cancer genomes that can be applied to prioritize putative non-coding driver regions in cancer. First, we aimed to develop a method that was applicable to entire genome, both coding and non-coding, rather than only a subset of regions. Second, we decided to incorporate information on evolution conservation in addition to mutation recurrence, and to determine what impact the inclusion of this information has on the regions identified. We developed a procedure for validating the performance of our scoring method that is based on the ability to identify known driver genes within coding regions. We also applied our method in a cancer type specific analysis to evaluate the possibility that some non-coding driver regions might be mutated in a cancer type specific manner.

\section{Results}

We have developed a scoring method, described in detail below, that identifies regions of the genome that are more frequently mutated compared to flanking regions (recurrence score) and that have mutations at bases that are more highly conserved (conservation score). We have applied this method to 1349 whole cancer genomes from a variety of cancer types (Additional file 1: Table S1) for $50 \mathrm{bp}$ windows spanning the entire human genome. Unlike previous efforts aimed at identifying non-coding driver mutations, which have usually focused on a limited set of non-coding regions (e.g. promoters, DNase I hypersensitive sites) we have applied our method in an unbiased manner to the entire genome, with the sole exception of regions where mappability is a concern. Here, we examine the characteristics and performance of our scores, as well as highlighting some promising candidate regions.

\section{Mutational processes in cancer genomes}

Our objective was to identify regions of the non-coding genome that are under positive selection during tumourogenesis. We searched for regions of the genome that are recurrently somatically mutated in cancer, a signal of positive selection. Although recurrent mutation may be a result of selection, it may also result from mutational processes acting on cancer genomes. There is considerable heterogeneity in mutation rates between different regions of the genome [9] as well as between different tumours (Additional file 2: Figure S1). To discover 
regions that are mutated more than would be expected from underlying mutational processes, we implemented a score that normalized for the mutation rate in flanking regions. This method can account for mutational processes that are constant over large portions of the genome, but may falsely identify portions of the genome that are particularly susceptible to mutation within a focused region. Because of the possibility that such focal mutational processes might contaminate regions identified by our scoring method, we additionally sought to understand mutational processes acting on whole cancer genomes for the purpose of flagging regions that are potential false positives.

\section{Identification of putative hypermutated regions}

We reasoned that regions of the genome with unusually high exposure to mutational processes would be expected to have a consistently elevated likelihood of mutation, whereas selection is expected to diminish once a driver has already been mutated. For example, gain of function mutations in oncogenes generally only need to occur once to confer driver activity, and often display mutual exclusivity with other mutations that have the same effects or that target the same pathway. Tumour suppressor genes are an exception, where two mutations may be required to confer driver activity. Thus, regions that are susceptible to mutation are more likely to sustain repeated mutations within the same region in the same tumour, while regions that are recurrently mutated due to selection are more likely to be mutated only once per tumour. In order to identify regions that may be recurrently mutated due to mutational processes rather than selection, we calculated the average number of mutations per patient for each region under consideration. We considered a region to be potentially hypermutated when the region had an average of 1.2 mutations per mutated patient or greater. We examined the prevalence of mutations within these putative hypermutated regions across tumour types. Several tumour types have an excess of mutations from hypermutated regions (Fig. 1) such as lymphomas
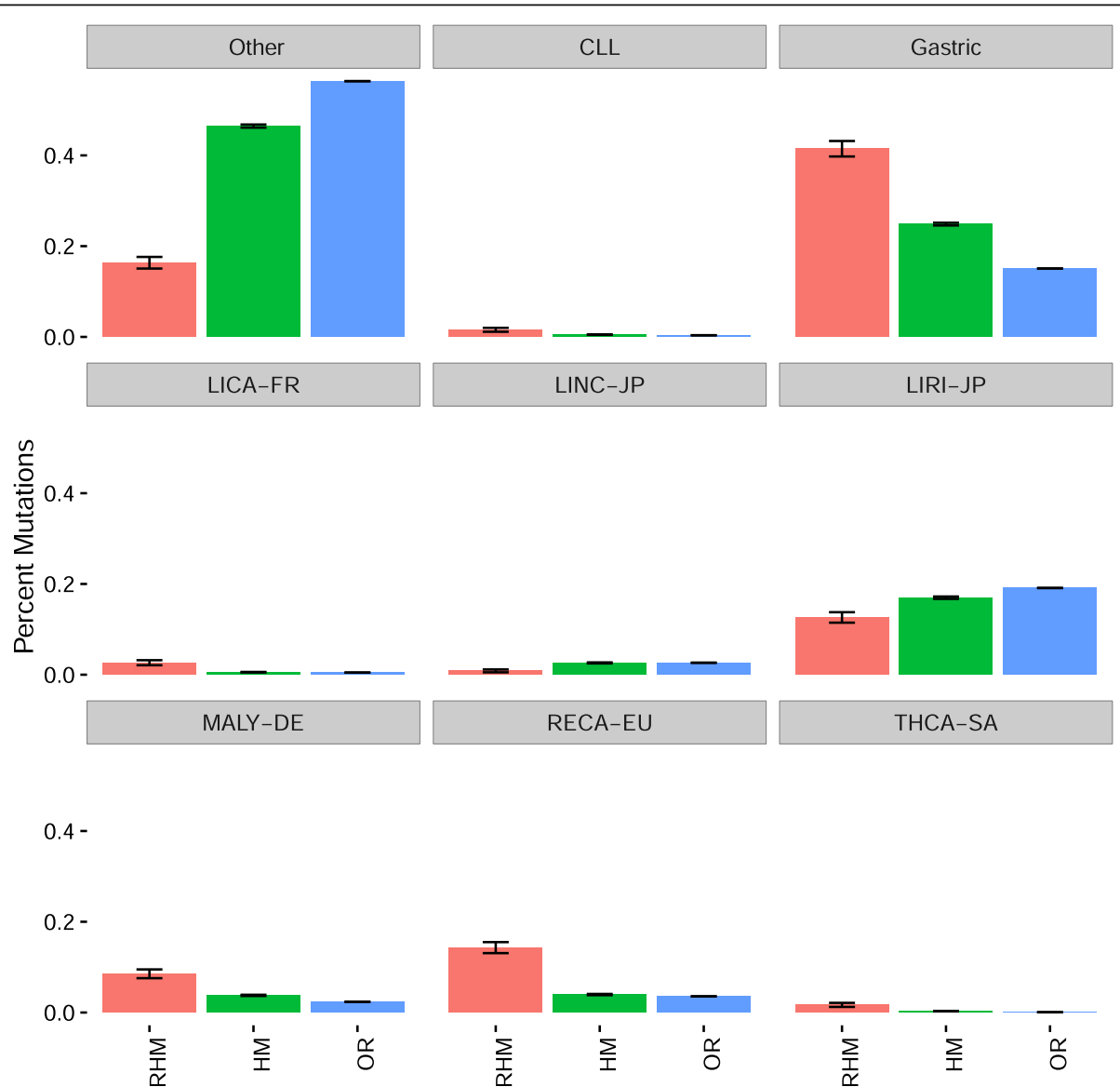

Fig. 1 For each of three categories: recurrent and hyper mutated regions (RHM, red, 832 total mutations), non-recurrent hypermutated regions (HM, green, 20958 total mutations), and other regions (OR, blue, 10713694 total mutations), we show the percent of mutations within region that belong to different cancer types. Malignant lymphoma has a disproportionate share of hypermutated regions, suggesting that our method of identifying hypermutated regions is capturing some regions that are targets of somatic hypermutation in this cohort. We define a region to be hypermutated when it has > 1.2 mutations per tumour, and to be recurrently mutated when it has a recurrence score greater than 10 
("MALY-DE") and renal cancers ("RECA-EU"). Several of the regions that we have identified as being hypermutated by this method lie in promoter regions and are primarily mutated in lymphoma, potentially suggesting that these regions are targets of somatic hypermutation rather than selection. Furthermore, some of these regions such as the promoter regions of $B C L 2$ and $M Y C$ have been identified as putative targets of selection in a previous analysis [26]. Analysis of mutational signatures within the putatively hypermutated regions that we identified did not identify any specific mutation process that could explain the pattern of base substitutions in these regions (Fig. 2), although it is possible that this mutational pattern is partially due to a process identified in CLL and lymphoma that is implicated with AID induced somatic hypermutation [27]. To evaluate the possible sensitivity of our method for identifying hypermutated regions to the specific threshold we use, we compared the classification of regions at a threshold of 1.2 with several other thresholds. For all values, $>97 \%$ of regions received the same designation

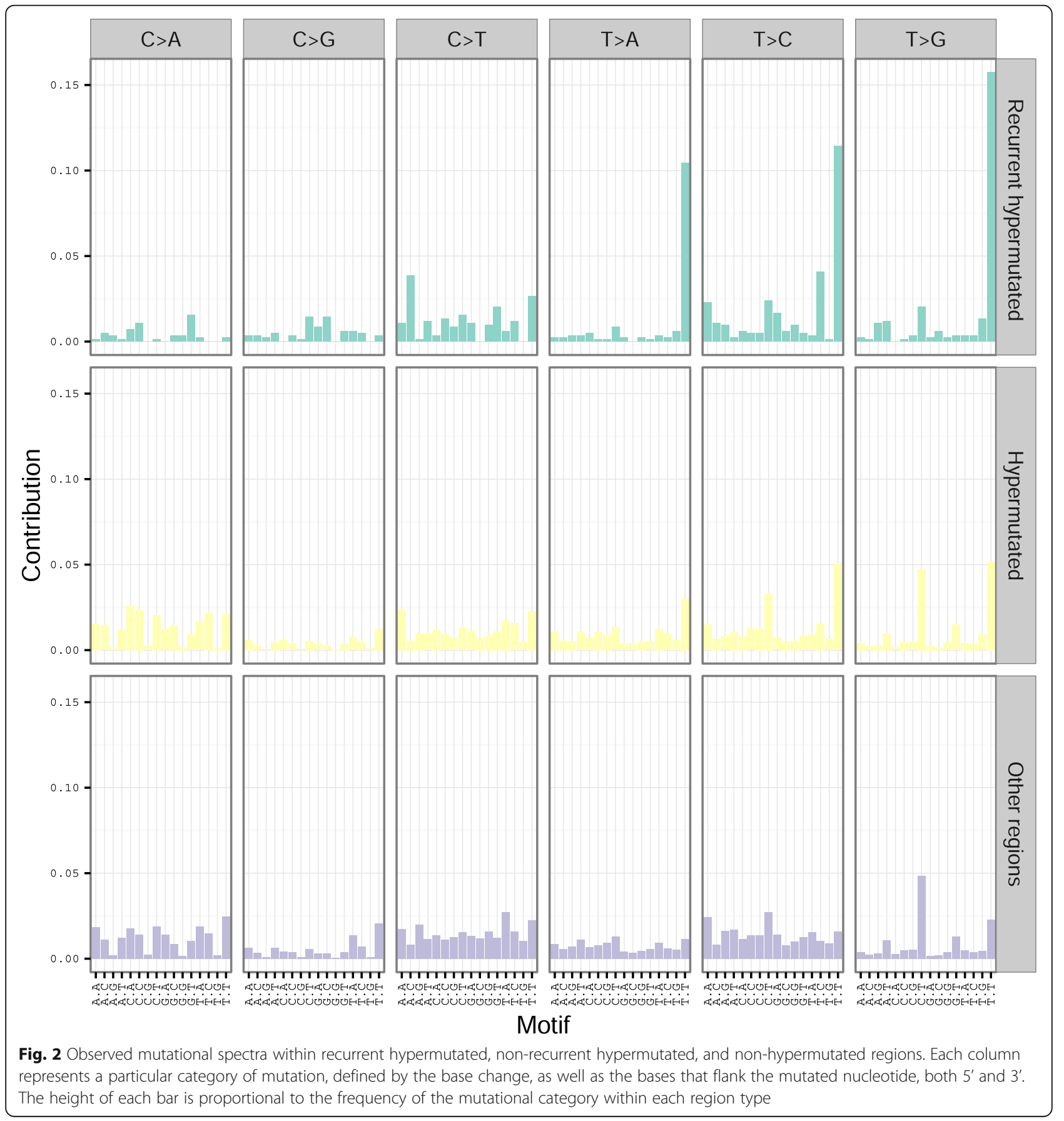


(hypermutated vs non-hypermutated) when compared to the 1.2 threshold. We therefore use the $>1.2$ threshold throughout the rest of our analysis.

\section{Mutational processes at CTCF binding sites}

In addition to the putatively hypermutated regions that we identified, we also observed that many recurrently mutated regions overlap regions with ChIP-seq evidence of CTCF binding (Fig. 3a, CTCF binding vs other regions $p=3.8 \times 10^{-18}$, CTCF DNase I hypersensitive vs other regions $p=2.08 \times 10^{-263}$, CTCF binding vs CTCF DNase I hypersensitive $\left.p=1.24 \times 10^{-46}\right)$. A recent analysis also identified an association between CTCF binding and recurrent mutation [20] potentially suggesting selection of these mutations, while other evidence from colorectal cancer by Katainen et al. suggests that CTCF binding sites may be subject to a unique mutational process which displays an excess of $\mathrm{T}>\mathrm{G}(\mathrm{A}>\mathrm{C})$ and $\mathrm{T}>\mathrm{C}(\mathrm{A}>\mathrm{G})$ mutations [28]. To discern whether the observed recurrence at CTCF binding sites in our dataset could result from a mutational process rather than selection, we compared the mutations at CTCF binding sites with the signature observed in Katainen et al. [28]. While CTCF binding sites in general do not show a signature similar to the one in [28] CTCF binding sites that we also identified as recurrent in our analysis display an excess of $\mathrm{T}>\mathrm{G}$ and $\mathrm{T}>\mathrm{C}$ mutations (Fig. $3 \mathrm{~b}$ ). When we examined specific recurrently mutated CTCF binding site that was also identified in [28] we found that the same bases within the binding site were recurrently mutated
(Additional file 2: Figure S2). This suggests that the recurrently mutated CTCF binding sites identified by our analysis are likely the result of the same process implicated in Katainen et al. [28]. CTCF binding sites that additionally have overlapping evidence of DNase I hypersensitivity in encode data display increased recurrence scores, consistent with the explanation that these mutations are the result of a mutational process related to DNA repair [29]. Many of the CTCF mutations in our sample come from a set of gastric cancer genomes, a cancer type not previously included by Katainen et al. Our analysis thus extends these observed patterns to this cancer type. Recent analyses have shown that transcription factor bound regions of the genome are subject to unique mutational processes and these mutations often preferentially target certain bases (e.g. G/C bases) [29, 30]. Our recurrence score correlates weakly with GC context (rank correlation 0.113) perhaps due to coding driver genes having high GC\% (Additional file 2: Figure S3). Regions with recurrence score $>10$ have comparable GC\% to regions with score $<$ 10 (Wilcoxon rank sum $p$-value $=0.81$ ).

\section{Pan-cancer prioritisation of non-coding mutations}

Having identified CTCF binding sites and regions with $>1.2$ mutations per tumour as regions that might be enriched for false positives, we next sought to identify regions that were likely to be under selection. We validated our prioritisation scores by considering exonic regions within our sample, because many large analyses have already identified known driver genes in protein

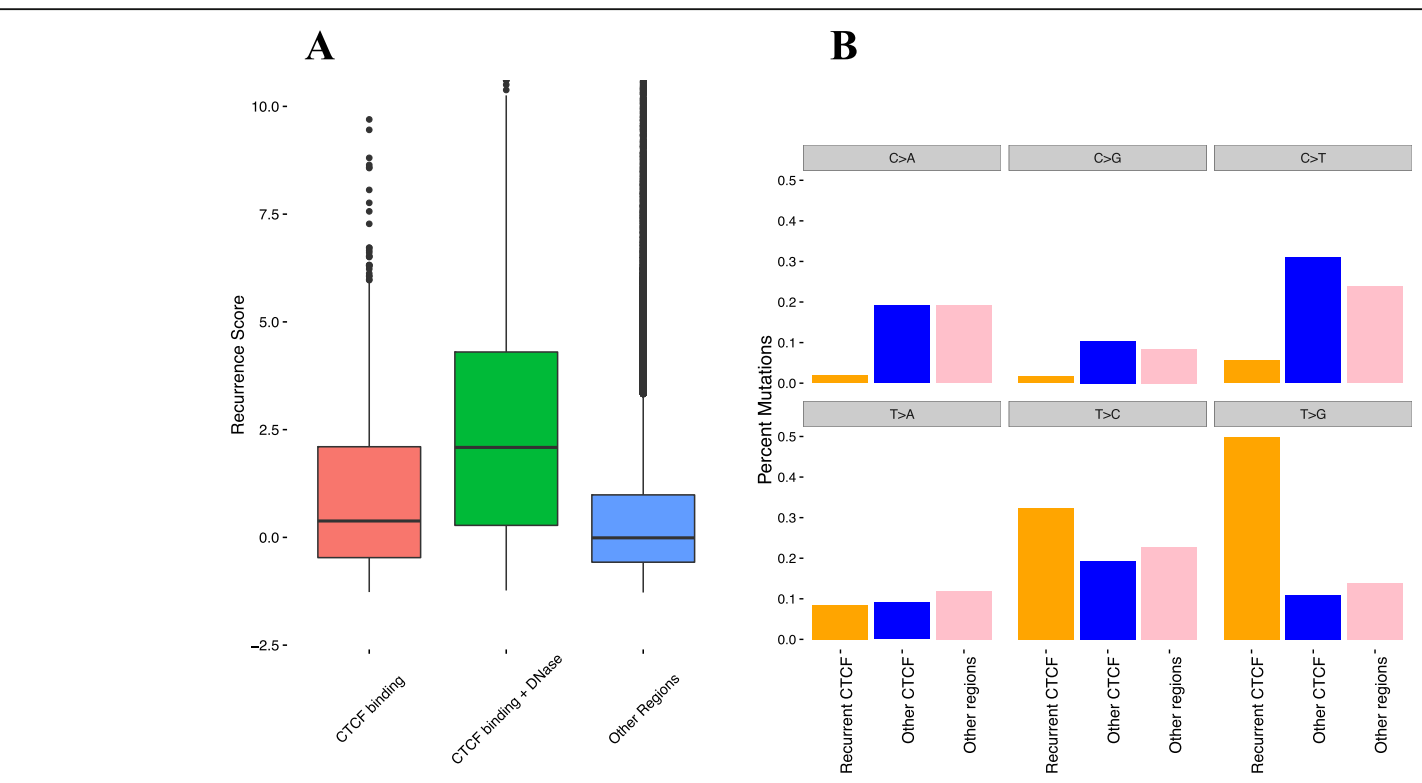

Fig. 3 (a) CTCF binding sites that overlap (green) and do not overlap (red) DNase I hypersensitive sites show a higher recurrence score compared to non-CTCF binding regions (blue); (b) We classified mutations as coming from recurrent CTCF binding sites (orange), non-recurrent CTCF binding sites (blue) and non-CTCF binding sites (pink). For each of these three categories, we give percentages indicating how many mutations from each category exhibit each of the six possible base changes. We define a CTCF binding site as recurrent when it has a recurrence score greater than 10 
coding regions. Our recurrence score $\left(p=3.8 \times 10^{-27}\right)$, conservation score $\left(p=1.32 \times 10^{-19}\right)$, and combined score $\left(p=3.22 \times 10^{-30}\right)$ were able to discriminate known driver genes within the set of all exonic regions (Fig. 4 a-c), suggesting that our method has reasonable effectiveness within this subset of the genome, despite the fact that we did not take advantage of annotations that are available for coding mutations (e.g. nonsynonymous vs synonymous mutations). We confirmed this by direct comparison of scores between driver and non-driver regions, as well as by simulation. To compare the known driver regions to a set of non-drivers of equal size, we resampled the non-driver exonic regions 10,000 times for each score, and compared the median score of the sampled non-drivers to the observed median of the known drivers. For all three scores, none of the 10,000 samples exceeded the median driver score (Fig. $4 \mathrm{~d}$-f). Several of the top scoring coding regions overlap well-known driver genes such as TP53 and KRAS. To investigate whether the inclusion of coding sequence within flanking regions had an impact on the regions identified, we also rescored each candidate region, this time excluding coding regions from the calculation of the flanking mutation rate. The regions identified were largely similar, with $94 \%$ of top regions in common between the two scoring methods. In order to assess whether the mutational counts are dominated by hypermutated samples, we recalculated the number of mutations in each $50 \mathrm{bp}$ window, excluding samples that are two standard deviations above the mean number of mutations. These counts are highly correlated $(r=0.88, p<0.0001)$ and this correlation is maintained when considering only regions that have greater than 5 mutations in the full dataset $(r=0.937, p<0.00001)$.

In addition to identifying known coding drivers, we also identified recurrently mutated non-coding regions, including both previously identified regions as well as novel regions (Fig. 5; Tables 1, 2, 3 and 4). We identified TERT (Additional file 2: Figure S4) and PLEKHS1 (Additional file 2: Figure S5) promoters as being recurrently mutated, consistent with previous analyses [21]. TERT appears in the top 50 regions genome-wide by recurrence (Table 1)

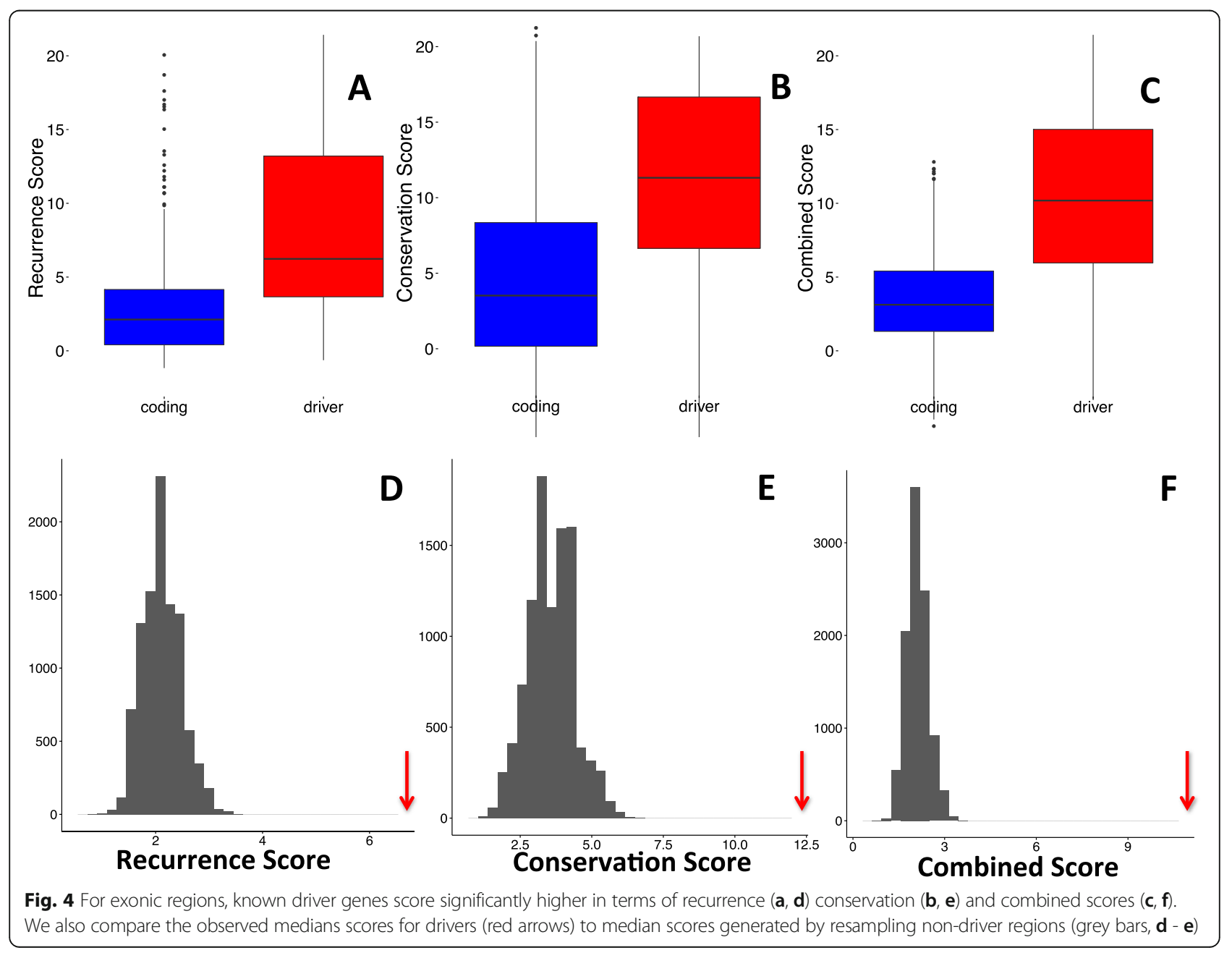




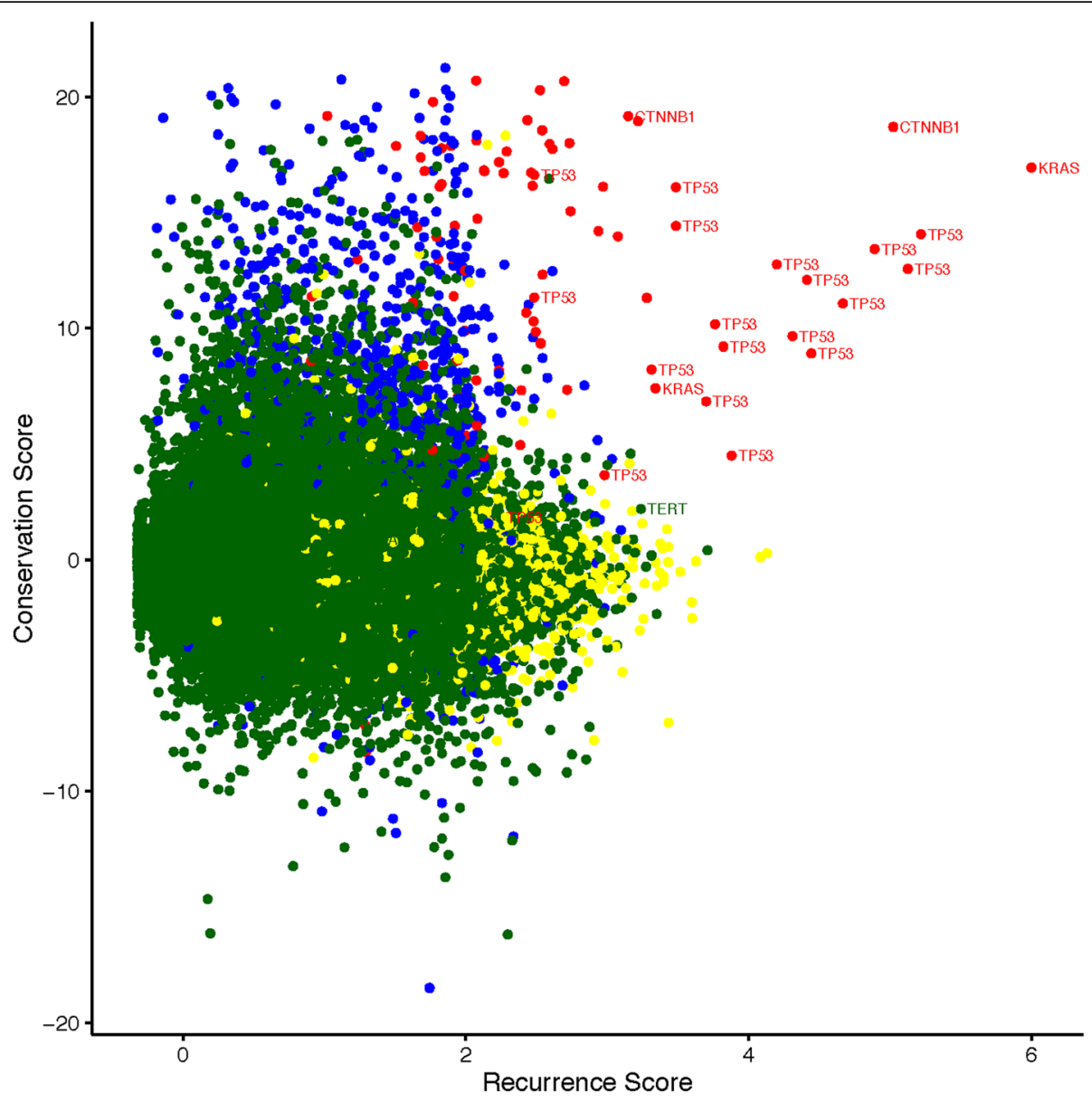

Fig. 5 Scatterplot of all regions mutated in more than two patients with conservation score on the vertical axis and Log (recurrence score +2 ) on the horizontal axis. The points are colored based on a classification of each region into one of four categories: coding, non-driver regions (blue), coding driver regions (red), non-coding, hypermutated regions (yellow), and non-coding non-hypermutated regions (green). Several known driver regions are also labelled

but not when ranked by the combined score (Table 3 ). One explanation for this is that in a genome-wide context, adding conservation will tend prioritise coding regions more highly, given the higher conservation of coding compared to non-coding regions. In support of this interpretation, Table 3 appears to be enriched for coding drivers relative to Table 1, while comparison of the top ten noncoding, non-hypermutated regions based on recurrence (Table 2) and combined score (Table 4) are highly similar. Despite the similarity of these lists, adding conservation does prioritise some interesting regions, including an intronic region that shows high conservation, as well as a conserved region of a miRNA. We discuss several candidate regions in more detail in the next section.

\section{Novel recurrent non-coding mutations}

Our method has highlighted several novel non-coding regions that may be selected for in cancer. Many highly recurrent regions are either known coding drivers or are regions that we have identified as hypermutated.
Although a region can be both hypermutated and selected, we focus on highlighting regions that are less likely to hypermutated. To demonstrate the types of novel regions identified by our analysis, we examined several regions that scored among the top regions in terms of both recurrence and conservation scores in our pan-cancer analysis.

The first region that we examined lies between the protein-coding gene MED16 and the small nuclear RNA RNU6-2 (Additional file 2: Figure S6). This regions lies within a DNase I hypersensitivity site and shows heavy transcription factor binding, suggestive of promoter activity or some other regulatory function. Each mutation within the region lies within a conserved sub-region of the window. No mutations fall within the unconserved regions surrounding this sub-region or within the nearby RNA gene, despite the fact that these latter regions make up the majority of the window. Driver mutations often displaying clustering within specific functional regions. The pattern observed in this region, with mutations clustered within a single conserved element, is potentially 
Table 1 Top 50 regions in terms of recurrence score identified by our method. We give the position of the region, number of genomes that are mutated within the region, the recurrence score, and a classification of the region based annotations and our method of identifying hypermutated regions. We also manually annotated each region by viewing in the UCSC genome browser

\begin{tabular}{|c|c|c|c|c|c|c|c|}
\hline Rank & Chr & Start & End & Mutated samples & Score & Automated annotation & Manual annotation \\
\hline 1 & chr12 & 25398250 & 25398300 & 256 & 399.9 & Driver & KRAS exon \\
\hline 2 & chr17 & 7577100 & 7577150 & 68 & 182.1 & Driver & TP53 exon \\
\hline 3 & chr17 & 7577500 & 7577550 & 62 & 165.7 & Driver & TP53 exon \\
\hline 4 & chr3 & 41266100 & 41266150 & 65 & 149.3 & Driver & CTNNB1 exon \\
\hline 5 & chr17 & 7578400 & 7578450 & 50 & 130.6 & Driver & TP53 exon \\
\hline 6 & chr17 & 7577550 & 7577600 & 41 & 103.9 & Driver & TP53 exon \\
\hline 7 & chr17 & 7578200 & 7578250 & 32 & 82.8 & Driver & TP53 exon \\
\hline 8 & chr17 & 7578250 & 7578300 & 31 & 80.1 & Driver & TP53 exon \\
\hline 9 & chr17 & 7577050 & 7577100 & 29 & 72.2 & Driver & TP53 exon \\
\hline 10 & chr17 & 7578500 & 7578550 & 26 & 64.4 & driver & TP53 exon \\
\hline 11 & chr10 & 96652800 & 96652850 & 14 & 60.0 & hotspot & non-coding \\
\hline 12 & chr12 & 6899300 & 6899350 & 3 & 57.1 & hotspot & CD4 intron \\
\hline 13 & chr17 & 7574000 & 7574050 & 19 & 46.2 & driver & TP53 exon \\
\hline 14 & chr17 & 7578450 & 7578500 & 18 & 43.5 & driver & TP53 exon \\
\hline 15 & chr17 & 7578350 & 7578400 & 17 & 40.9 & driver & TP53 exon \\
\hline 16 & chr3 & 195892250 & 195892300 & 18 & 38.7 & non-coding & non-coding \\
\hline 17 & chr17 & 7577000 & 7577050 & 14 & 38.3 & driver & TP53 exon \\
\hline 18 & chr12 & 64749950 & 64750000 & 7 & 35.5 & hotspot & C12orf56 intron \\
\hline 19 & chr13 & 50016900 & 50016950 & 8 & 34.5 & hotspot & CAB39L intron \\
\hline 20 & chr11 & 63881800 & 63881850 & 9 & 34.4 & hotspot & FLRT1 intron \\
\hline 21 & chr15 & 64857000 & 64857050 & 9 & 31.6 & hotspot & ZNF609 intron \\
\hline 22 & chr17 & 7578150 & 7578200 & 13 & 30.6 & driver & TP53 exon \\
\hline 23 & chr17 & 7578550 & 7578600 & 13 & 30.5 & driver & TP53 splice site \\
\hline 24 & chr16 & 88383450 & 88383500 & 7 & 28.9 & hotspot & Non-coding / TF binding \\
\hline 25 & chr14 & 24895100 & 24895150 & 11 & 28.8 & hotspot & Non-coding / TF binding \\
\hline 26 & chr17 & 79389900 & 79389950 & 9 & 28.8 & hotspot & $B A H C C 1$ intron \\
\hline 27 & chr17 & 17424850 & 17424900 & 7 & 28.5 & hotspot & PEMT intron \\
\hline 28 & chr22 & 46697350 & 46697400 & 5 & 27.8 & hotspot & GTSE1 intron \\
\hline 29 & chr8 & 30717550 & 30717600 & 7 & 27.8 & hotspot & TEX15 exon-intron border \\
\hline 30 & chr7 & 76949650 & 76949700 & 6 & 27.6 & hotspot & GSAP intron \\
\hline 31 & chr14 & 74239050 & 74239100 & 8 & 27.2 & hotspot & ELMSAN1 intron \\
\hline 32 & chr4 & 819750 & 819800 & 6 & 27.0 & hotspot & CPLX1 intron \\
\hline 33 & chr16 & 81908550 & 81908600 & 7 & 26.4 & hotspot & PLCG2 intron \\
\hline 34 & chr4 & 39684550 & 39684600 & 10 & 26.4 & non-coding & non-coding \\
\hline 35 & chr22 & 39962000 & 39962050 & 6 & 26.2 & hotspot & non-coding \\
\hline 36 & chr12 & 25380250 & 25380300 & 20 & 26.1 & driver & KRAS exon \\
\hline 37 & chr3 & 43746400 & 43746450 & 11 & 25.4 & non-coding & $A B H D 5$ intron \\
\hline 38 & chr17 & 7579300 & 7579350 & 10 & 25.4 & driver & TP53 exon \\
\hline 39 & chr9 & 21971100 & 21971150 & 12 & 24.5 & driver & CDKN2A exon \\
\hline 40 & chr8 & 9921850 & 9921900 & 12 & 24.3 & non-coding & MRSA intron \\
\hline 41 & chr11 & 70764100 & 70764150 & 6 & 24.1 & hotspot & SHANK2 intron \\
\hline 42 & chr19 & 12597300 & 12597350 & 9 & 23.8 & hotspot & ZNF709 intron \\
\hline
\end{tabular}


Table 1 Top 50 regions in terms of recurrence score identified by our method. We give the position of the region, number of genomes that are mutated within the region, the recurrence score, and a classification of the region based annotations and our method of identifying hypermutated regions. We also manually annotated each region by viewing in the UCSC genome browser (Continued)

\begin{tabular}{llllllll}
\hline 43 & chr17 & 49455750 & 49455800 & 10 & 23.6 & hotspot & non-coding \\
44 & chr5 & 1295200 & 1295250 & 14 & 23.4 & non-coding & TERT promoter \\
45 & chr7 & 151591800 & 151591850 & 6 & 23.2 & hotspot & non-coding \\
46 & chr21 & 44524450 & 44524500 & 9 & 22.9 & driver & U2AF1 exon \\
47 & chr1 & 45914900 & 45914950 & 7 & 22.7 & hotspot & TESK2 intron \\
48 & chr8 & 29901300 & 29901350 & 9 & 22.4 & non-coding & non-coding \\
49 & chr7 & 606050 & 606100 & 7 & 22.0 & hotspot & PRKAR1B intron \\
50 & chr2 & 49173750 & 49173800 & 27 & 22.0 & non-coding & CTCF binding \\
\hline
\end{tabular}

suggestive of driver activity. Given the evidence for transcription factor binding in this region, one possibility is that this conserved sub-region is a motif associated with protein binding. Although mutations at this locus are focused within this conserved subregion, the mutations are spread throughout the subregion, not focused at any single nucleotide, and do not always show consistent base changes in the cases where the mutations do occur at the same nucleotide. Assuming that these mutations are in fact targeting some kind of binding motif, the relatively even distribution of mutations without consistent base changes possibly suggests that these mutations are disrupting a binding motif as opposed to a creating a novel motif. To assess the possibility that these mutations may alter protein-binding motifs at the site, we searched the reference sequence of the mutated region for possible matches with known motifs. We identified matches with the transcription factors FOXL1, NKX3-1, and MEF2A. We also searched for matches when the reference sequence is replaced with several of the mutants observed in our dataset. In the case of MEF2A both mutations we tested reduced the maximum similarity score from 13.7 to 5.7 and 0.92 , suggesting that the mutations observed in this region may be disruptive to this motif (Additional file 2: Figure S7).

The second region that we highlight is deep within the intron of the gene GPR126 (Additional file 2: Figure S8). This region shows high levels of conservation, and the mutations observed is region occur exclusively at two base positions. All mutations within this region are entirely mutually exclusive, and there are no other mutations within this region other than at these two positions. This pattern of mutation is similar to that initially observed at mutations in the TERT promoter, and is suggestive of driver activity. These mutations also occur at the same positions within a motif (GAAC) as mutations in the PLEKHS1 promoter, potentially suggesting a common process is occurring at these two loci. These mutations lie far from any exon-intron boundaries, ruling out the possibility that they affect donor or acceptor sites. This region overlaps a DNase I hypersensitive site, potentially suggesting that this region contains on intronic regulatory elements. We identified motifs matching the transcription factors FOXL1, POU2F2, FOXA1, and FOXP2 overlapping this region. We did not notice a consistent pattern in the effects of the observed mutations on motif occurrence.

Table 2 Top ten non-coding, non-hypermutated regions in terms of recurrence score

\begin{tabular}{lllllll}
\hline rank & chr & start & end & samples mutated & score & manual annotation \\
\hline 1 & chr3 & 195892250 & 195892300 & 18 & 38.7 & non-coding \\
2 & chr4 & 39684550 & 39684600 & 10 & 26.4 & non-coding \\
3 & chr3 & 43746400 & 43746450 & 11 & 25.4 & ABHD5 intron \\
4 & chr8 & 9921850 & 9921900 & 12 & 24.3 & MSRA intron \\
5 & chr5 & 1295200 & 1295250 & 14 & 23.4 & TERT promoter \\
6 & chr8 & 29901300 & 29901350 & 9 & 22.4 & non-coding \\
7 & chr2 & 49173750 & 49173800 & 27 & 22.0 & CTCF binding \\
8 & chr8 & 70576150 & 70576200 & 21 & 21.8 & CTCF binding \\
9 & chr19 & 893450 & 893500 & 9 & 21.6 & MED16 promoter \\
10 & chr2 & 47359300 & 47359350 & 8 & 21.0 & C2orf61 intron \\
\hline
\end{tabular}


Table 3 Top 50 regions in terms of combined score identified by our method. We give the position of the region, number of genomes that are mutated within the region, the combined score, and a classification of the region based annotations and our method of identifying hypermutated regions. We also manually annotated each region by viewing in the UCSC genome browser

\begin{tabular}{|c|c|c|c|c|c|c|c|}
\hline rank & chr & Start & End & Mutated samples & Score & Automated annotation & Manual annotation \\
\hline 1 & chr12 & 25398250 & 25398300 & 256 & 208.4 & driver & KRAS exon \\
\hline 2 & chr17 & 7577100 & 7577150 & 68 & 98.1 & driver & TP53 exon \\
\hline 3 & chr17 & 7577500 & 7577550 & 62 & 89.1 & driver & TP53 exon \\
\hline 4 & chr3 & 41266100 & 41266150 & 65 & 84.0 & driver & CTNNB1 exon \\
\hline 5 & chr17 & 7578400 & 7578450 & 50 & 72.0 & driver & TP53 exon \\
\hline 6 & chr17 & 7577550 & 7577600 & 41 & 57.5 & driver & TP53 exon \\
\hline 7 & chr17 & 7578250 & 7578300 & 31 & 46.1 & driver & TP53 exon \\
\hline 8 & chr17 & 7578200 & 7578250 & 32 & 45.8 & driver & TP53 exon \\
\hline 9 & chr17 & 7577050 & 7577100 & 29 & 40.9 & driver & TP53 exon \\
\hline 10 & chr17 & 7578500 & 7578550 & 26 & 38.6 & driver & TP53 exon \\
\hline 11 & chr10 & 96652800 & 96652850 & 14 & 30.1 & hotspot & Non-coding \\
\hline 12 & chr12 & 6899300 & 6899350 & 3 & 28.6 & hotspot & CD4 intron \\
\hline 13 & chr17 & 7578450 & 7578500 & 18 & 26.4 & driver & TP53 exon \\
\hline 14 & chr17 & 7578350 & 7578400 & 17 & 25.5 & driver & TP53 exon \\
\hline 15 & chr17 & 7574000 & 7574050 & 19 & 25.4 & driver & TP53 exon \\
\hline 16 & chr17 & 7578550 & 7578600 & 13 & 23.3 & driver & TP53 exon \\
\hline 17 & chr17 & 7577000 & 7577050 & 14 & 22.6 & driver & TP53 exon \\
\hline 18 & chr17 & 7578150 & 7578200 & 13 & 22.5 & driver & TP53 exon \\
\hline 19 & chr21 & 44524450 & 44524500 & 9 & 20.9 & driver & TP53 exon \\
\hline 20 & chr3 & 41266050 & 41266100 & 10 & 20.2 & driver & CTNNB1 exon \\
\hline 21 & chr3 & 195892250 & 195892300 & 18 & 19.5 & non-coding & Non-coding \\
\hline 22 & chr9 & 21971100 & 21971150 & 12 & 17.9 & driver & CDKN2A exon \\
\hline 23 & chr12 & 64749950 & 64750000 & 7 & 17.7 & hotspot & C12orf56 intron \\
\hline 24 & chr17 & 7579300 & 7579350 & 10 & 16.8 & driver & TP53 exon \\
\hline 25 & chr2 & 198266800 & 198266850 & 9 & 16.8 & driver & SF3B1 exon \\
\hline 26 & chr12 & 25380250 & 25380300 & 20 & 16.8 & driver & KRAS exon \\
\hline 27 & chr18 & 48591900 & 48591950 & 11 & 16.8 & driver & SMAD4 exon \\
\hline 28 & chr3 & 178936050 & 178936100 & 9 & 16.7 & driver & PIK3CA exon \\
\hline 29 & chr11 & 63881800 & 63881850 & 9 & 16.3 & hotspot & FLRT1 intron \\
\hline 30 & chr13 & 50016900 & 50016950 & 8 & 16.0 & hotspot & CAB39L intron \\
\hline 31 & chr19 & 11134250 & 11134300 & 6 & 15.7 & driver & SMARCA4 exon \\
\hline 32 & chr15 & 64857000 & 64857050 & 9 & 15.5 & hotspot & ZNF609 intron \\
\hline 33 & chr20 & 57484400 & 57484450 & 13 & 15.5 & driver & GNAS exon \\
\hline 34 & chr16 & 3786700 & 3786750 & 5 & 15.4 & driver & CREBBP exon \\
\hline 35 & chr17 & 17424850 & 17424900 & 7 & 14.9 & hotspot & PEMT intron \\
\hline 36 & chr14 & 24895100 & 24895150 & 11 & 14.7 & hotspot & Non-coding / TF binding \\
\hline 37 & chr18 & 48575150 & 48575200 & 7 & 14.7 & driver & SMAD4 exon \\
\hline 38 & chr18 & 48604750 & 48604800 & 7 & 14.6 & driver & SMAD4 exon \\
\hline 39 & chr19 & 11132500 & 11132550 & 5 & 14.6 & driver & SMARCA4 exon \\
\hline 40 & chr17 & 79389900 & 79389950 & 9 & 14.3 & hotspot & BAHCCl exon \\
\hline 41 & chr18 & 48591800 & 48591850 & 8 & 14.2 & driver & SMAD4 exon \\
\hline 42 & chr3 & 178952050 & 178952100 & 7 & 14.2 & driver & PIK3CA exon \\
\hline
\end{tabular}


Table 3 Top 50 regions in terms of combined score identified by our method. We give the position of the region, number of genomes that are mutated within the region, the combined score, and a classification of the region based annotations and our method of identifying hypermutated regions. We also manually annotated each region by viewing in the UCSC genome browser (Continued)

\begin{tabular}{llllllll}
\hline 43 & chr7 & 76949650 & 76949700 & 6 & 14.0 & hotspot & GSAP intron \\
44 & chr14 & 74239050 & 74239100 & 8 & 13.9 & hotspot & ELMSAN1 intron \\
45 & chr17 & 56408600 & 56408650 & 5 & 13.9 & non-coding & MIR142 non-coding \\
46 & chr22 & 46697350 & 46697400 & 5 & 13.6 & hotspot & GTSE1 intron \\
47 & chr8 & 30717550 & 30717600 & 7 & 13.4 & hotspot & TEX15 exon-intron border \\
48 & chr10 & 89692900 & 89692950 & 3 & 13.3 & driver & PTEN exon \\
49 & chr17 & 7577600 & 7577650 & 5 & 13.3 & driver & TP53 splice site \\
50 & chr4 & 819750 & 819800 & 6 & 13.2 & hotspot & CPLX1 intron \\
\hline
\end{tabular}

We additionally identified recurrent mutations at highly conserved positions overlapping the miRNA MIR142 (Additional file 2: Figure S9). These mutations are spread throughout the region, and occur exclusively in lymphoma samples, suggesting that this region may be a target of somatic hypermutation. Puente et al. also identified recurrent mutations near MIR142 in CLL, which they attribute to somatic hypermutation [22]. Despite the fact that this region may be a target of hypermutation rather than selection, the appearance of this region within the top ten non-coding, non-hypermutated regions in terms of combined score (Table 4) but not recurrence score (Table 2) suggests that conservation can highlight regions that are highly conserved but have lower recurrence. All but one of the mutations observed in our dataset overlap the mature microRNA hsa-miR-142-5p based on the miRBase [31] sequence (Additional file 2: Figure S10), suggesting that these mutations may have an impact of the ability of the mircoRNA to bind target mRNAs. This creates the possibility that this region is a target of both hypermutation and selection. As a result, it may be useful to use both scores separately to nominate regions with different characteristics. Finally, we highlight a recurrently mutated region in an intron in the gene MSRA
(Additional file 2: Figure S11). Similar to several of the other regions highlighted, this region is mutated predominantly at two base positions, which in this case occur at neighbouring positions. We additionally identified motifs that are potential matches for transcription factors SOX9 and SRY overlapping this region. We did not notice a consistent pattern in the effects of the observed mutations on motif occurrence.

\section{Cancer type specific analysis}

So far, we have focused on regions that are mutated in multiple cancer types. To investigate if some non-coding driver mutations are mutated primarily in one or a few cancer types only, we applied our scoring method independently to each cancer type in the dataset with more than 75 whole genomes. Consistent with our pan-cancer analysis, when we applied our method to the exonic regions of specific cancer types, we again identified many known cancer genes (Fig. 6). Several of the genes that we identified are particularly prominent in cancer types in which they are known to be highly mutated, such as VHL in renal cancer, PIK3CA in breast cancer, TP53 in ovarian cancer, $S M A D 4$ in esophageal and gastric cancer, and $K R A S$ in pancreatic cancer.

Table 4 Top ten non-coding, non-hypermutated regions in terms of combined score

\begin{tabular}{lllllll}
\hline rank & chr & start & end & samples mutated & score & manual annotation \\
\hline 1 & chr3 & 195892250 & 195892300 & 18 & 38.7 & non-coding \\
2 & chr4 & 396845550 & 39684600 & 10 & 26.4 & non-coding \\
3 & chr3 & 43746400 & 43746450 & 11 & 25.4 & ABHD5 intron \\
4 & chr8 & 9921850 & 9921900 & 12 & 24.3 & MSRA intron \\
5 & chr5 & 1295200 & 1295250 & 14 & 23.4 & TERT promoter \\
6 & chr8 & 29901300 & 29901350 & 9 & 22.4 & non-coding \\
7 & chr2 & 49173750 & 49173800 & 27 & 22.0 & CTCF binding \\
8 & chr19 & 893450 & 893500 & 9 & 21.6 & MED16 promoter \\
9 & chr6 & 142706200 & 142706250 & 9 & 18.0 & GPR126 intron \\
10 & chr17 & 56408600 & 56408650 & 5 & 11.3 & MIR142 \\
\hline
\end{tabular}




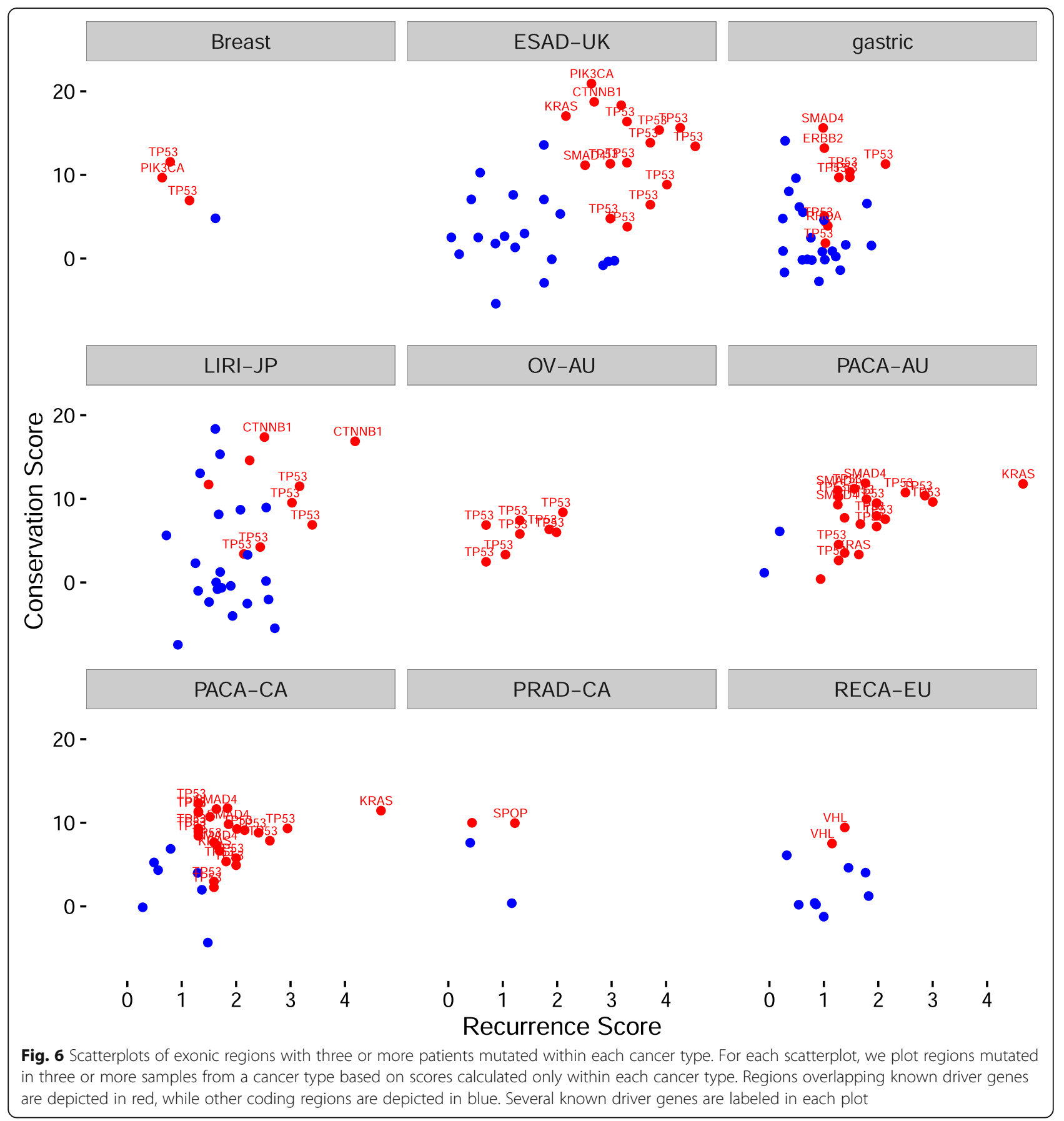

\section{Cancer type specific non-coding mutations}

In addition to the regions identified in our pan-cancer analysis, we also identified non-coding regions that are recurrently mutated in individual cancer types (Additional file 1: Tables S2 and S3). We identified recurrent mutations within an intron of the PRIM2 gene (Additional file 2: Figure S12) specifically in renal cancer. These mutations occurred at two bases in a mutually exclusive manner, and exclusively in renal cancer samples. We identified motifs matching the transcription factors FOXL1, BRCA1,
FOXH1, FOXP1, PRDM1, TCF7L2, ZNF236, IRF1, STAT3, and FOXP2 overlapping this region. Two mutant sequences we tested had maximum scores of 11.1 compared to -0.8 for matches to FOXP2 (Additional file 2: Figure S13). We also identified recurrent mutations within an intron of $R A D 51 B$ in several breast cancer samples (Additional file 2: Figure S14). RAD51B is a DNA repair gene involved in homologous recombination [32]. We identified motifs matching the transcription factors FOXC1, MZF1_5-13, MAFF, MAFK, EGR1, ESR2, 
GATA2, GATA3, and THAP1 overlapping this region. We did not notice a consistent pattern in the effects of the observed mutations on motif occurrence. Given the importance of this repair pathway in breast cancer, this region may warrant further study in this cancer type. Within the regions prioritised by the combined score, we also identified several extremely highly conserved regions that are recurrently mutated in the LIRI-JP cohort (liver cancer), including non-coding regions of the genes BCL11A, $B C L 6$, and PAX5 (Additional file 1: Table S3).

\section{Discussion}

As is the case in the analysis of coding mutations, we have found that mutational heterogeneity is a critical factor that impacts the identification of non-coding driver regions in cancer. Our initial analysis revealed that several promising candidate regions, some of which have been suggested in the literature as potential driver regions, may actually be recurrently mutated primarily due to focal mutational processes rather than selection. We have found potential evidence of an AID associated somatic hypermutation signature as well as a recenty identified process which targets CTCF binding sites [28] as prominent local mutational processes. In addition, we have proposed methods for identifying and filtering out these putatively hypermutated regions, allowing greater focus on regions for which we believe the evidence favouring positive selection is stronger. Using the exome to validate our scoring method, we showed that all three scores can differentiate known drivers from other coding regions. We also identified several known driver genes that display a mutation pattern across cancer types consistent with expectations.

In addition to using recurrence as previous studies have, we included conservation as part of the prioritization scores. We have shown that the conservation score can separate known coding drivers from non-drivers. Conservation may also be useful in the analysis of non-coding mutations, both to increase confidence that recurrent non-coding mutations have the potential to impact function, as well as to highlight non-coding regions that may have lower recurrence but driver potential due to higher conservation. The combined score also appears to outperform the recurrence score alone in terms of distinguishing known driver regions from other exonic regions, suggesting that conservation provides valuable information in addition to recurrence, although this may be more difficult to interpret within the context of non-coding mutations, given that non-coding regions are generally less well conserved as a whole compared to coding regions. The generally low conservation observed in non-coding regions sugggests that functional non-coding mutations might not necessarily always occur at conserved positions. Thus, it is useful to consider recurrent mutations, even if they are not at highly conserved positions. Using a measure such as the combined score may also highlight regions that have moderate recurrence but which are highly conserved. These regions would be good candidates for more "hill-like" drivers [8]. As a result, we believe that using both recurrence and a combined score that incorporates recurrence and conservation to prioritise regions that may have different properties is a promising strategy. It is also worth noting that more complex ways of combining these scores might yield additional benefits. We have averaged the scores, after normalizing to make the scores roughly comparable, but other transformations might also produce insights.

Within these genomes, we also identified several novel recurrently mutated regions. In addition to the novel recurrent regions we identified in a pan-cancer analysis, we also identifed several novel non-coding regions that appear to be cancer type specific, some of which have high frequencies in the cancer types in which they occur. These regions, as well as other regions that score highly within our framework, may be good targets for future analyses of non-coding somatic mutations in cancer. Although the methods used here can not definitively establish a mutation as a driver, further investigation of non-coding mutations using these and other methods may reveal new non-coding driver mutations. These drivers may have important implications for cancer therapy if they are directly targetable by drugs or involved in the regulation of pathways that are targetable. Non-coding mutations such as TERT promoter mutations [33] have been associated with clinical outcomes, as have mutational processes in cancer [34-36]. We have highlighted regions that have an excess of mutations in cancer genomes. These regions may lead to important insights that may have clinical implications if they are either under selection or indicative of underlying mutational processes.

\section{Conclusions}

We have developed a novel method for the identification of putative driver regions in cancer, which is applicable to both coding and non-coding regions. We have shown that this method performs well at identifying prominent coding and non-coding regions that are known or highly suspected to play a role in cancer. Unlike previous attempts to identify recurrently mutated non-coding regions, we apply our method to the entire genome to identify novel non-coding regions mutational hotspots. We also highlight recurrently mutated regions that may have resulted from increased exposure to mutational process rather than selection, some of which have been identified previously as targets of selection.

\section{Methods}

In order to identify recurrently mutated non-coding regions that are potential targets of somatic selection during the development of cancer, we devised a scoring 
system to prioritise regions of the genome based on signatures that are indicative of selection. In the context of coding mutations, driver genes are known to be recurrently mutated above background mutation rates and also show a pattern of enrichment for functional mutations (e.g. stop-gain, non-synonymous) compared to mutations that are less likely to be function (e.g. synonymous mutations). Applying similar principles to non-coding regions, we developed two scores, one that is designed to detect regions that are recurrently mutated, and a second designed to detect regions that have mutations at conserved bases, working on the hypothesis that conserved positions are more likely to be functional. We then applied these scores, as well as a combined score, to a set of over 1300 cancer whole genomes.

\section{Whole genome mutation data}

We assembled a set of pre-called somatic mutations from three sources: release 18 of ICGC [37], data from Alexandrov et al. [27], and the supplemental materials of Wang et al. [38]. Some of these sources contain data from both whole exome and whole genome sequencing. We only analyzed mutations annotated as coming from whole genome sequencing. To avoid the possibility of duplicated samples, in cases where the same tumour type was included in ICGC and the data from Alexandrov et al. we included data from only one source. The distribution of samples across tumour types and data sources is summarized in Additional file 1: Table S1. After filtering out samples lacking sufficient numbers of mutations, we were left with a total of 1349 samples for our final analysis.

\section{Annotation data}

We used the UCSC genome browser [39, 40] to obtain various annotation files, including dbSNP and COSMIC variants, information on gene models, conservation, mappability, and epigenetic data.

\section{Software}

We processed genomic data using bedtools v2.25.0 [41] and conducted statistical analysis and data manipulation in R 3.2.3 [42].

\section{Processing mutation data}

We annotated all data to human reference genome version hg19. Preliminary analysis revealed several frequent mutations that overlap known germline SNPs, suggestive of the possibility that these mutations are not truly somatic. We removed from consideration mutations that occur at the same genomic coordinate as a known dbSNP entry, unless that genomic position was also annotated as mutated in COSMIC (cancer.sanger.ac.uk) [5]. After filtering out known dbSNP entries, we also excluded tumour samples with fewer than 1000 total mutations from further analysis. For dbSNP variants, we used build 142 of dbSNP. dbSNP and COSMIC variant locations were obtained in bed format from the UCSC Table Browser [39].

\section{Annotating and filtering genomic regions}

We divided the reference hg19 genome into $50 \mathrm{bp}$, nonoverlapping windows using the bedtools makewindows command. We mapped mutations to each window, and calculated the mean 100-way PhyloP score as well as the mean 35 bp uniqueness (a measure of sequence mappability) across mutations that fell within the window. We excluded from further consideration any window that had a mean mappability of its overlapping mutations that was less than 0.5 , as well as any window that was mutated in fewer than 3 patients (because these regions lack sufficient mutations to be considered recurrent).

\section{Calculation of recurrence score}

For each region that met our filtering criteria (candidate regions), we calculated a recurrence score representing the level of enrichment of the region with mutations compared to the mutation rate within the region of the genome flanking the region under consideration. For each candidate region, we formed a flanking region, which included the region of the genome that was within $0.5 \mathrm{Mb}$ of the region on either side, truncated at chromosome ends. We removed bases within the flanking region that had mappability less than 0.5 . We calculated a flanking mutation rate for each candidate region by dividing the number of mutations in our set of whole genomes that overlap valid flanking base positions by the number of valid bases within the flanking region. We calculated a raw mutation score (Equation 1) by dividing the rate (mutations per nucleotide) in the candidate region by the flanking mutation rate. We normalized this raw mutation score by subtracting the median score from all candidate regions and dividing each score by the median absolute deviation (mad) over all candidates (Equation 2). We initially planned to perform the normalization by flanking mutation rate separately for each tumour sample, but this was not feasible due to the sparsity of mutations in some samples. Equations for the raw and normalized recurrence scores are:

$$
\text { raw score }=\frac{T / T_{0}}{(L+R) /\left(L_{0}+R_{0}\right)}
$$

Where $\mathrm{T}$ is the number of mutations observed in the target region, $\mathrm{T}_{0}$ is the length of the target region, $\mathrm{L}$ and $\mathrm{R}$ are the number of mutations in the left and right flanking regions of the target region, and $\mathrm{L}_{0}$ and $\mathrm{R}_{0}$ are the lengths of the left and right flanking regions. 


$$
\text { normalized score }=\frac{\text { raw score }- \text { median }(\text { raw score })}{\operatorname{mad}(\text { raw score })}
$$

\section{Calculation of conservation score}

For each candidate region, we also calculated a conservation score. Our strategy was to use a basepair level measure of conservation, and average across mutations to score a region based on conservation. We chose the PhyloP score [43] calculated on a 100-way species tree, which is available from the UCSC genome browser. PhyloP scores as implemented in the UCSC Genome Browser are negative log base $10 \mathrm{p}$-values for a likelihood ratio test against the null hypothesis of neutral evolution. The scores are positive when the test indicates that the nucleotide evolves more slowly (i.e. is conserved) and negative in the case that it evolves more quickly (acceleration). For each mutation, we mapped PhyloP scores of the base position at which the mutation occurred. Within each candidate region, we took the mean of the PhyloP scores for each mutation within the region as a raw conservation score. Similar to our recurrence score, we normalized this raw conservation score by subtracting the median score and dividing by the median absolute deviation.

\section{Calculation of combined score}

For each candidate region, we calculated the combined score as the simple average of normalized recurrence and conservation scores.

\section{Statistical analysis}

For comparison of scores in different classes of regions, we used Mann-Whitney tests, as implemented in R. we also performed simulations to compare the median scores of known driver regions to non-driver exonic regions. We repeated sampled with replacement 10,000 samples of non-driver regions with size equal to the number of candidate regions overlapping known driver regions, took the median score for each sample, and compared to the observed median for known driver genes.

\section{Collation of known driver genes}

Driver genes were collated in humans by combining gene lists from two previously published lists of driver genes from Vogelstein et al. and Lawrence et al. [4, 6]. Gene names were taken from table S2A of Vogelstein et al. [4] and from Additional file 1: Table S2 from Lawrence et al. [6]. These gene names were entered into the UCSC Table Browser [39] to obtain hg19 coordinates for the coding exons of these genes, which were mapped to mutations using bedtools [41]. We considered a region to be a known driver if it overlapped a coding exon of a gene listed in either publication. In total, we constructed a set of 308 driver genes.

\section{Threshold sensitivity analysis}

For all regions with greater than 2 mutations, we classified the region as either hypermutated or non-hypermuated based on whether the mutations per mutated sample in that region exceed a threshold, where exceeding the threshold resulted in classification as a hypermutated region. We classified regions in this way for thresholds of 1.1, 1.3, 1.3 and 1.5, and compared these classifications to a threshold of 1.2. For each comparison, we calculated the percent of regions that had the same classification (both hypermutated or both non-hypermutated) in the comparison.

\section{Transcription factor binding motif analysis}

We obtained position weight matrices for human transcription factors using the "JASPAR2014" package in R [44], and searched for matches using the "searchSeq" function from the "TFBSTools" package [45] with default settings. We also selected recurrent mutations occurring within candidate regions and searched against the mutated sequence for transcription factors that matched the reference.

\section{Additional files}

\section{Additional file 1: Table S1. The number of samples with 1000 or more valid mutations included in our final analysis, as well as information about tumour type and original publication for each sample. For the ICGC samples we give ICGC project codes and use this to categorise tumour type throughout this work. Although some project codes imply the same tumour type (e.g. LICA-FR and LINC-JP are both liver cancers) we treat these separately in case these cohorts might have different properties, either technical or biological. Table S2: Top ten non-coding, non- hypermutated regions in terms of recurrence score within each cancer type. Table S3: Top ten non-coding, non-hypermutated regions in terms of combined score within each cancer type. (PDF $196 \mathrm{~kb}$ )}

Additional file 2: Figure S1. Log10 of total mutations per genome, ordered by median mutations within each tumour type. Figure S2: For comparison, we show the location of mutations (black arrows) within a recurrent CTCF binding site that was highlighted in a previous analysis [28]. Figure S3: We show recurrence score (plotted as log(score + 2)) plotted against GC content. Regions with mutations per patient $>1.2$ are in orange, with recurrence score $>10$ and mutations per patient $<=1.2$ in black, and all others in purple. Figure S4: Recurrent TERT promoter mutations identified in our data set. The mutations occur at one of the previously identified bases, generating a de novo ETS binding site. Figure S5: PLEKHS1 recurrently mutated region that has previously been identified. We identify mutations at the same base position as previous analyses. Figure S6: UCSC browser image depicting a recurrently mutated region identified by our method. Mutations are depicted by black arrows. This region is flanked on the left by the gene MED16. Figure S7: Sequence logo depicting the MEF2A motif. Text above the logo is the reference sequence observed within the recurrently mutated region in the MED16 promoter. Mutated positions are depicted in red. Figure S8: UCSC browser image of a second recurrently mutated region identified by our method. Mutations are depicted by black arrows. Figure S9: Recurrently mutated region overlapping the miRNA MIR142. The region is highly conserved, as suggested by its inclusion among the top non-coding regions based 
on combined score. Figure S10: MIR142 reference aligned with the sequence of mature microRNA has-miR142-5p. Mutated positions are depicted in red. Figure S11: Recurrently mutation overlapping an intron of the gene MSRA. The mutations occur primarily at two neighbouring bases. Figure S12: UCSC browser image of a recurrently mutated region overlapping an intron of the gene PRIM2. Figure S13: Sequence logo depicting the FOXP2 motif. Text above the logo is the reference sequence observed within the recurrently mutated region in the PRIM2 intron. Mutated positions are depicted in red. Figure S14: UCSC browser image depicting a recurrently mutated region in an intron of the DNA repair gene RAD51B. This region is mutated specifically in breast cancer. (PDF $4081 \mathrm{~kb}$ )

\section{Acknowledgements}

The results published here are in whole or part based upon data generated by the International Cancer Genome Consortium. We would like to thank the Irish Centre for High End Computing (https://www.ichec.ie/) for the use of HPC infrastructure.

\section{Funding}

This work was supported by the European Commission (FP7-PEOPLE-2013IEF - 627027 to SJF), and the Irish Research Council Bioinformatics and System Biology Ph.D. Programme (http://www.research.ie/ to SWP), and the Irish Cancer Society CCRC BREAST-PREDICT (CCRC13GAL).

\section{Availability of data and material}

Not applicable.

\section{Authors' contributions}

SJF conceived and supervised the study. SWP conducted all bioinformatic and statistical analysis. Both authors drafted the manuscript.

\section{Competing interests}

The authors declare that they have no competing interests.

\section{Consent for publication}

Not applicable.

\section{Ethics approval and consent to participate}

No ethics approval was required for the study, which is based on publicly available data.

\section{Author details}

'School of Biomolecular and Biomedical Science, Conway Institute of Biomolecular and Biomedical Research, University College Dublin, Dublin, Ireland. ${ }^{2}$ School of Biomolecular and Biomedical Science, Conway Institute of Biomolecular and Biomedical Research, University College Dublin, Belfield, Dublin 4, Ireland.

Received: 5 August 2016 Accepted: 14 December 2016 Published online: 05 January 2017

\section{References}

1. Cancer Genome Atlas Research N, Weinstein JN, Collisson EA, Mills GB, Shaw KR, Ozenberger BA, Ellrott K, Shmulevich I, Sander C, Stuart JM. The Cancer Genome Atlas Pan-Cancer analysis project. Nat Genet. 2013;45(10):1113-20.

2. Garraway LA, Lander ES. Lessons from the cancer genome. Cell. 2013;153(1):17-37.

3. Stratton MR, Campbell PJ, Futreal PA. The cancer genome. Nature. 2009:458(7239):719-24.

4. Vogelstein B, Papadopoulos N, Velculescu VE, Zhou S, Diaz Jr LA, Kinzler KW. Cancer genome landscapes. Science. 2013;339(6127):1546-58.

5. Forbes SA, Beare D, Gunasekaran P, Leung K, Bindal N, Boutselakis H, Ding M, Bamford S, Cole C, Ward S, et al. COSMIC: exploring the world's knowledge of somatic mutations in human cancer. Nucleic Acids Res. 2015:43(Database issue):D805-811.

6. Lawrence MS, Stojanov P, Mermel CH, Robinson JT, Garraway LA, Golub TR, Meyerson M, Gabriel SB, Lander ES, Getz G. Discovery and saturation analysis of cancer genes across 21 tumour types. Nature. 2014;505(7484):495-501.
7. Tamborero D, Gonzalez-Perez A, Perez-Llamas C, Deu-Pons J, Kandoth C, Reimand J, Lawrence MS, Getz G, Bader GD, Ding L, et al. Comprehensive identification of mutational cancer driver genes across 12 tumor types. Sci Rep. 2013;3:2650.

8. Wood LD, Parsons DW, Jones S, Lin J, Sjoblom T, Leary RJ, Shen D, Boca SM, Barber T, Ptak J, et al. The genomic landscapes of human breast and colorectal cancers. Science. 2007;318(5853):1108-13.

9. Lawrence MS, Stojanov P, Polak P, Kryukov GV, Cibulskis K, Sivachenko A, Carter SL, Stewart C, Mermel CH, Roberts SA, et al. Mutational heterogeneity in cancer and the search for new cancer-associated genes. Nature. 2013;499(7457):214-8.

10. Gonzalez-Perez A, Lopez-Bigas N. Functional impact bias reveals cancer drivers. Nucleic Acids Res. 2012;40(21):e169.

11. Tamborero D, Gonzalez-Perez A, Lopez-Bigas N. OncodriveCLUST: exploiting the positional clustering of somatic mutations to identify cancer genes. Bioinformatics. 2013:29(18):2238-44.

12. Stehr $H$, Jang $S H$, Duarte JM, Wierling $C$, Lehrach $H$, Lappe M, Lange BM. The structural impact of cancer-associated missense mutations in oncogenes and tumor suppressors. Mol Cancer. 2011;10:54.

13. Piraino SW, Furney SJ. Beyond the exome: the role of non-coding somatic mutations in cancer. Ann Oncol. 2016;27(2):240-8.

14. Huang FW, Hodis E, Xu MJ, Kryukov GV, Chin L, Garraway LA. Highly Recurrent TERT Promoter Mutations in Human Melanoma. Science. 2013;339(6122):957-9.

15. Horn S, Figl A, Rachakonda PS, Fischer C, Sucker A, Gast A, Kadel S, Moll I, Nagore E, Hemminki K, et al. TERT promoter mutations in familial and sporadic melanoma. Science. 2013;339(6122):959-61.

16. Mathelier A, Lefebvre C, Zhang AW, Arenillas DJ, Ding J, Wasserman WW, Shah SP. Cis-regulatory somatic mutations and gene-expression alteration in B-cell lymphomas. Genome Biol. 2015;16:84.

17. Poulos RC, Thoms JA, Shah A, Beck D, Pimanda JE, Wong JW. Systematic Screening of Promoter Regions Pinpoints Functional Cis-Regulatory Mutations in a Cutaneous Melanoma Genome. Mol Cancer Res. 2015;13(8):1218-26.

18. Fredriksson NJ, Ny L, Nilsson JA, Larsson E. Systematic analysis of noncoding somatic mutations and gene expression alterations across 14 tumor types. Nat Genet. 2014:46(12):1258-63.

19. Fu Y, Liu Z, Lou S, Bedford J, Mu XJ, Yip KY, Khurana E, Gerstein M. FunSeq2: a framework for prioritizing noncoding regulatory variants in cancer. Genome Biol. 2014;15(10):480.

20. Lochovsky L, Zhang J, Fu Y, Khurana E, Gerstein M. LARVA: an integrative framework for large-scale analysis of recurrent variants in noncoding annotations. Nucleic Acids Res. 2015;43(17):8123-34.

21. Weinhold N, Jacobsen A, Schultz N, Sander C, Lee W. Genome-wide analysis of noncoding regulatory mutations in cancer. Nat Genet. 2014;46(11):1160-5.

22. Puente XS, Bea S, Valdes-Mas R, Villamor N, Gutierrez-Abril J, Martin-Subero Jl, Munar M, Rubio-Perez C, Jares P, Aymerich M, et al. Non-coding recurrent mutations in chronic lymphocytic leukaemia. Nature. 2015;526(7574):519-24.

23. Mularoni L, Sabarinathan R, Deu-Pons J, Gonzalez-Perez A, Lopez-Bigas N. OncodriveFML: a general framework to identify coding and non-coding regions with cancer driver mutations. Genome Biol. 2016;17(1):128.

24. Melton C, Reuter JA, Spacek DV, Snyder M. Recurrent somatic mutations in regulatory regions of human cancer genomes. Nat Genet. 2015;47(7):710-6.

25. Nik-Zainal S, Davies H, Staaf J, Ramakrishna M, Glodzik D, Zou X, Martincorena I, Alexandrov LB, Martin S, Wedge DC, et al. Landscape of somatic mutations in 560 breast cancer whole-genome sequences. Nature. 2016;534(7605):47-54.

26. Smith KS, Yadav VK, Pedersen BS, Shaknovich R, Geraci MW, Pollard KS, De S. Signatures of accelerated somatic evolution in gene promoters in multiple cancer types. Nucleic Acids Res. 2015;43(11):5307-17.

27. Alexandrov LB, Nik-Zainal S, Wedge DC, Campbell PJ, Stratton MR. Deciphering signatures of mutational processes operative in human cancer. Cell Rep. 2013;3(1):246-59.

28. Katainen R, Dave K, Pitkanen E, Palin K, Kivioja T, Valimaki N, Gylfe AE, Ristolainen H, Hanninen UA, Cajuso T, et al. CTCF/cohesin-binding sites are frequently mutated in cancer. Nat Genet. 2015;47(7):818-21.

29. Perera D, Poulos RC, Shah A, Beck D, Pimanda JE, Wong JW. Differential DNA repair underlies mutation hotspots at active promoters in cancer genomes. Nature. 2016;532(7598):259-63.

30. Sabarinathan R, Mularoni L, Deu-Pons J, Gonzalez-Perez A, Lopez-Bigas N. Nucleotide excision repair is impaired by binding of transcription factors to DNA. Nature. 2016;532(7598):264-7.

31. Griffiths-Jones S, Grocock RJ, van Dongen S, Bateman A, Enright AJ. miRBase: microRNA sequences, targets and gene nomenclature. Nucleic Acids Res. 2006;34(Database issue):D140-144. 
32. Takata M, Sasaki MS, Sonoda E, Fukushima T, Morrison C, Albala JS, Swagemakers SM, Kanaar R, Thompson LH, Takeda S. The Rad51 paralog Rad51B promotes homologous recombinational repair. Mol Cell Biol. 2000;20(17):6476-82.

33. Borah S, Xi L, Zaug AJ, Powell NM, Dancik GM, Cohen SB, Costello JC, Theodorescu D, Cech TR. Cancer. TERT promoter mutations and telomerase reactivation in urothelial cancer. Science. 2015;347(6225):1006-10.

34. Le DT, Uram JN, Wang H, Bartlett BR, Kemberling H, Eyring AD, Skora AD, Luber BS, Azad NS, Laheru D, et al. PD-1 Blockade in Tumors with Mismatch-Repair Deficiency. N Engl J Med. 2015;372(26):2509-20.

35. Snyder A, Makarov V, Merghoub T, Yuan J, Zaretsky JM, Desrichard A, Walsh LA Postow MA, Wong P, Ho TS, et al. Genetic basis for clinical response to CTLA-4 blockade in melanoma. N Engl J Med. 2014;371(23):2189-99.

36. Waddell N, Pajic M, Patch AM, Chang DK, Kassahn KS, Bailey P, Johns AL, Miller D, Nones K, Quek K, et al. Whole genomes redefine the mutational landscape of pancreatic cancer. Nature. 2015;518(7540):495-501.

37. Zhang J, Baran J, Cros A, Guberman JM, Haider S, Hsu J, Liang Y, Rivkin E, Wang J, Whitty B, et al. International Cancer Genome Consortium Data Portal-a one-stop shop for cancer genomics data. Database (Oxford). 2011;2011:bar026.

38. Wang K, Yuen ST, Xu J, Lee SP, Yan HH, Shi ST, Siu HC, Deng S, Chu KM, Law $\mathrm{S}$, et al. Whole-genome sequencing and comprehensive molecular profiling identify new driver mutations in gastric cancer. Nat Genet. 2014;46(6):573-82.

39. Karolchik D, Hinrichs AS, Furey TS, Roskin KM, Sugnet CW, Haussler D, Kent WJ. The UCSC Table Browser data retrieval tool. Nucleic Acids Res. 2004;32(Database issue):D493-496.

40. Kent WJ, Sugnet CW, Furey TS, Roskin KM, Pringle TH, Zahler AM, Haussler D. The human genome browser at UCSC. Genome Res. 2002;12(6):996-1006.

41. Quinlan AR, Hall IM. BEDTools: a flexible suite of utilities for comparing genomic features. Bioinformatics. 2010;26(6):841-2.

42. Team RDC. R: A language and environment for statistica computing. Vienna: R Foundation for Statistical Computing; 2010.

43. Pollard KS, Hubisz MJ, Rosenbloom KR, Siepel A. Detection of nonneutral substitution rates on mammalian phylogenies. Genome Res. 2010;20(1):110-21.

44. Mathelier A, Zhao X, Zhang AW, Parcy F, Worsley-Hunt R, Arenillas DJ, Buchman S, Chen CY, Chou A, lenasescu H, et al. JASPAR 2014: an extensively expanded and updated open-access database of transcription factor binding profiles. Nucleic Acids Res. 2014;42(Database issue):D142-147.

45. Tan G, Lenhard B. TFBSTools: an R/bioconductor package for transcription factor binding site analysis. Bioinformatics. 2016;32(10):1555-6.

\section{Submit your next manuscript to BioMed Central and we will help you at every step:}

- We accept pre-submission inquiries

- Our selector tool helps you to find the most relevant journal

- We provide round the clock customer support

- Convenient online submission

- Thorough peer review

- Inclusion in PubMed and all major indexing services

- Maximum visibility for your research

Submit your manuscript at www.biomedcentral.com/submit 